\title{
Verhaltenstherapie
}

R. Lieb

M. Mastaler

H.-U. Wittchen

Max-Planck-Institut für Psychiatrie, Klinische Psychologie und Epidemiologie, München

\section{Gibt es somatoforme Störungen bei Jugendlichen und jungen Erwachse- nen? Erste epidemiologische Befunde der Untersuchung einer bevölke- rungsrepräsentativen Stichprobe}

\author{
Are There Somatoform Disorders in Adolescents \\ and Young Adults? First Epidemiological Findings Based on a \\ Representative Population Sample
}

\section{Schlüsselwörter}

Somatoforme Störungen: Epidemiologie, Prävalenz, Komorbidität, Beeinträchtigung

\section{Zusammenfassung}

Auf der Grundlage der Basisuntersuchung einer epidemiologischen prospektiven Verlaufsstudie (1995-1999) an 3021 Personen im Alter zwischen 14 und 24 Jahren werden epidemiologische Befunde zur Häufigkeit von somatoformen Beschwerden und somatoformen Syndromen/Störungen bei Jugendlichen und jungen Erwachsenen berichtet. Die Jugendlichen und jungen Erwachsenen wurden mit Hilfe des M-CIDI, einem standardisierten Interview zur Erfassung psychischer Symptome, Syndrome und Störungen nach dem DSM-IV, befragt. Die Ergebnisse der ersten Untersuchung zeigen, daß $50 \%$ der Jugendlichen einmal in ihrem bisherigen Leben unter einem somatoformen Symptom litten. Junge Frauen berichten häufiger von somatoformen Beschwerden als junge Männer (61 vs. 40\%). Die im DSM-IV operationalisierten Kriterien einer somatoformen Störung werden nur von wenigen Jugendlichen und jungen Erwachsenen (2,7\%) erfüllt. Die Prävalenz erhöht sich jedoch, wenn man nicht ausschließlich voll ausgeprägte somatoforme Störungen, sondern zusätzlich unterschwellige Syndrome in die Betrachtung einschließt: Hier berichten etwa $11 \%$ der Jugendlichen von somatoformen Syndromen. Somatoforme Störungen/Syndrome zeigen sich häufig im Verbund mit anderen psychischen Störungen, wobei die Komorbidität mit dem Alter zunimmt. Wie unsere Analysen ergaben, berichten Jugendliche und junge Erwachsene mit somatoformen Störungen, aber auch solche mit unterschwelligen Syndromen, vermehrt Beeinträchtigungen in verschiedenen sozialen Rollenbereichen und der Arbeitsproduktivität.

\section{Key Words}

Somatoform disorders: epidemiology, prevalence, comorbidity, impairments

\section{Summary}

As part of a longitudinal study, prevalence findings of somatoform symptoms, syndromes and disorders are presented for a random sample of 3021 respondents aged 14 to 24 years. The response rate was $71 \%$. Assessment was made using the computer-assisted Munich-Composite International Interview (M-CIDI). Findings of the first part of the study revealed that $50 \%$ (men : $40 \%$; women: $61 \%$ ) of the sample once had a somatoform symptom in their life. Threshold somatoform disorders were rare with $2.7 \%$. However, when including subthreshold somatoform syndromes $(11 \%)$, the lifetime prevalence of any somatoform disorder/syndrome was $13 \%$. Somatoform disorders and syndromes are often comorbid with other mental disorders, and comorbidity rises with age. Further, they are associated with disabilities and impairments in social and work domains. 


\section{Einleitung}

Der Begriff «Somatoforme Störungen» beschreibt Beschwerdenbilder mit körperlichen Symptomen, die nicht hinreichend durch einen körperlichen Befund erklärbar sind. Allgemein wird vermutet, daß somatoforme Beschwerden in der Bevölkerung häufig vorkommen und daß ihnen aufgrund der durch sie verursachten Behandlungskosten und Beeinträchtigungen eine hohe gesundheitspolitische Bedeutung zukommt. Der Begriff bzw. die Gruppe der somatoformen Störungen wurde 1980 mit der 3. Auflage des DSM (DSM-III) [APA, 1980] eingeführt und findet sich seit der 10. Revision auch in der ICD (ICD-10) [WHO, 1991; 1993]. Obwohl in den letzten Jahren das Forschungsinteresse gegenüber dieser Störungsgruppe zunahm, sind nach wie vor grundlegende Aspekte wie etwa die diagnostisch-klassifikatorische (nosologische) Konzeptualisierung somatoformer Störungen, deren reliable und valide Erfassung, die differentialdiagnostische Abklärung, Fragen nach dem Beginn und dem typischen Verlauf und auch die ätiologischen sowie pathogenetischen Mechanismen nur in Ansätzen geklärt. Nicht zuletzt aufgrund der angeführten Probleme bestehen noch große Unsicherheiten über die tatsächliche Häufigkeit und den Verlauf somatoformer Störungen und über die mit ihnen assoziierten Risikofaktoren und Komplikationen. Abgesehen von methodisch unbefriedigenden klinischen Kohortenstudien, die meistens keine klinisch gesicherte Diagnostik beinhalten, liegen nur wenige Allgemeinbevölkerungsstudien älteren Datums (DSM-IIIKriterien) vor, die eine sehr niedrige Prävalenz von 1\% für die Somatisierungsstörung ermittelt haben (Epidemiological Catchment Area Program, ECA [Regier et al., 1988; 1993], Münchner Follow-up-Studie, MFS [Wittchen et al., 1992]).

Nach dem reformulierten trennschärferen DSM-IV umfaßt die Gruppe der somatoformen Störungen die «Somatisierungsstörung», «Undifferenzierte Somatoforme Störung», «Konversionsstörung», «Schmerzstörung», «Hypochondrie» und die «Körperdysmorphe Störung». Die Somatisierungsstörung kann durch eine große Anzahl (DSM-III [APA, 1980]: ein Minimum von 13 Symptomen; DSM-IV [APA, 1994]: ein Minimum von 8 Symptomen aus 4 unterschiedlichen Symptomgruppen) unterschiedlicher somatoformer Beschwerden charakterisiert werden. Die Befunde der ECAStudien und der MFS, in welchen die Auftretenshäufigkeit von psychischen Störungen unter Anwendung von standardisierten Interviews (Diagnostic Interview Schedule [Robins et al., 1991]) erhoben wurde, kommen übereinstimmend zu dem Ergebnis, daß dieses so eng definierte Störungsbild in der Allgemeinbevölkerung eher selten vorkommt (ECA: 0,1\%, MFS: 0,8\%). Angesichts dieser niedrigen Prävalenzraten, die im wesentlichen durch die enge Definition der Somatisierungsstörung bedingt sein dürften, und angesichts der Beobachtung, daß in der klinischen Praxis somatoforme Beschwerden gravierend häufiger wahrgenommen werden [Kellner, 1985; Rief und Hiller, 1992], wurden von einigen Arbeitsgrup- pen die operationalen Kriterien dieser Störung als zu eng und für den klinischen Alltag unbrauchbar bewertet (Deighton und Nicol, 1985; Escobar et al., 1987b; 1989). Als klinisch sinnvolle Alternative wurde von Escobar et al. [1987a] der sogenannte Somatic Symptom Index SSI4,6 vorgeschlagen, nach welchem von einer klinisch bedeutsamen Symptomatik bereits dann gesprochen werden kann, wenn Männer 4 und Frauen 6 somatoforme Symptome aufweisen. Mehrere Studien konnten zeigen, daß die Symptomatik des SSI4,6 zu ähnlichen sozialen und beruflichen Beeinträchtigungen führt wie das Vollbild der Somatisierungsstörung [Escobar und Canino, 1989; Escobar et al., 1989; Katon et al., 1991]. Nach den ECABefunden liegt die Prävalenz des SSI4,6 im US-amerikanischen Raum bei etwa 4\% (Los Angeles) [Escobar et al., 1989]. Für den deutschsprachigen Raum sind neben einigen klinischen Studien, die auf eine Häufigkeit in klinischen Stichproben von etwa 13-25\% hinweisen [Rief et al., 1992; Fydrich und Schmitz, 1994], derzeit keine epidemiologischen Befunde über die Prävalenz des SSI4,6 in der Allgemeinbevölkerung verfügbar.

Noch spärlicher fällt die epidemiologische Befundlage bei den restlichen somatoformen Störungen aus, da keine Daten bei den erwähnten epidemiologischen Studien ermittelt wurden. Hinsichtlich der Prävalenz der Hypochondrie liegen - allerdings nicht mittels standardisierter diagnostisch-klassifikatorischer Verfahren erhobene - Angaben zwischen 4 und 14\% vor [Viederman, 1993]. Befunde der WHO-Multicenter-Studie [Üstun und Sartorius, 1995], in welcher die Häufigkeit von ICD-10 Diagnosen in Allgemeinarztpraxen mittels des Composite Diagnostic Interview (CIDI) [WHO 1992] erfaßt wurde, verweisen jedoch auf weit niedrigere Häufigkeitsraten: Hier wurde - allerdings nur auf diese spezielle Inanspruchnahmepopulation bezogen - für die Hypochondrie eine Häufigkeitsrate von 0,8\% (Querschnitt) ermittelt. Für «Schmerzstörung», «Konversionsstörung» und «Körperdysmorphe Störung» liegen, abgesehen von groben Schätzungen - so führt die APA (DSM-IV) [APA, 1994] Prävalenzschätzungen von $10-15 \%$ für die Schmerzstörung und $0,003 \%$ für die Konversionsstörung an -, nach unserem Wissen überhaupt keine epidemiologisch reliablen Angaben über ihre Häufigkeit in der Allgemeinbevölkerung vor.

Häufig wird die Vermutung angestellt, daß die Entwicklung von somatoformen Beschwerden und Störungen im Kindesund Jugendalter einsetzt [Tomasson et al., 1991; Bass und Murphy, 1995]. Die wenigen, mit ganz verschiedenen Instrumenten bestimmten und auf unterschiedlichen Störungskonzeptionen beruhenden Prävalenzangaben bewegen sich je nach Altersgruppe und erfaßter Symptomatik zwischen 1,1 und 14\% [Pfeiffer et al., 1997].

Speziell bei der Untersuchung der Häufigkeit somatoformer Störungen im Kindes- und Jugendalter stellt sich zusätzlich die Frage nach a) der Validität der in der ICD-10 und dem DSM-IV aufgezeigten Diagnosekriterien für diese Altersgruppe und b) der Gültigkeit entsprechender Beurteilungsin- 
strumente. Schon bei der Betrachtung der im DSM-IV vorgegebenen Symptome fällt auf, daß bestimmte Symptome vor der Pubertät überhaupt nicht auftreten können (z.B. schmerzhafte Menstruation), so daß die Brauchbarkeit der definierten Schwellenwerte für Kinder und Jugendliche zu bezweifeln ist. Die DSM-IV-Kriterien verlangen außerdem zur Diagnosestellung einer somatoformen Störung in der Regel eine gewisse Persistenz/Chronifizierung der Symptomatik (länger als 6 Monate, Symptomatik führte zu einer klinischen Beeinträchtigung). Auch hier kann hinterfragt werden, ob durch die Vorgabe eines solchen Beschwerdezeitraumes nicht von vornherein Symptombilder ausgeschlossen werden, die möglicherweise nicht für Erwachsene, wohl aber für jüngere Altersstufen klinisch diagnostisch relevant sind bzw. klinisch bedeutsame Vorläuferstadien der Entwicklung einer somatoformen Störung darstellen.

In dem vorliegenden Beitrag berichten wir über erste Ergebnisse einer an einer repräsentativen Stichprobe von 3021 14-24jährigen Jugendlichen und jungen Erwachsenen durchgeführten prospektiven epidemiologischen Längsschnittuntersuchung, in der neben weiteren psychischen Störungen auch das Vorliegen von somatoformen Beschwerden und somatoformen Störungen nach dem DSM-IV erhoben wurde. Dabei versuchen wir folgende Fragen zu beantworten:

1. Wie häufig sind somatoforme Beschwerden (Symptomebene) bei Jugendlichen und jungen Erwachsenen, und welches sind die am häufigsten vorkommenden Beschwerden?

2. Wie häufig liegen in dieser Altersgruppe somatoforme Störungen und Syndrome (diagnostische Ebene) vor?

3. In welchem Alter treten somatoforme Störungen zum ersten Mal auf?

4. Treten somatoforme Störungen im Verbund mit anderen psychischen Störungen auf (Komorbidität)?

5. Wie beeinträchtigend sind somatoforme Störungen in dieser Altersgruppe?

\section{Methodik}

\section{Studie und Design}

Die Daten wurden im Rahmen der ersten Untersuchungswelle der Early Developmental Stages of Psychopathology Study (EDSP) erhoben. Sie ist eine prospektive epidemiologische Verlaufsuntersuchung (1995-1999, 3 Untersuchungswellen) an einer repräsentativen Stichprobe Jugendlicher und junger Erwachsener mit dem Ziel der Bestimmung von Prävalenz, Risikofaktoren, Komorbidität, Frühstadien und Entwicklungsverlauf von psychischen Störungen (eine detaillierte Beschreibung des methodischen Vorgehens findet sich bei Wittchen et al. [1998]).

\section{Stichprobe}

Befragt wurde eine Zufallsstichprobe von 14-24jährigen Jugendlichen und jungen Erwachsenen in München-Stadt und -Land. Nach einem Zufallsverfahren wurden 19944809 Personen dieser Altersgruppe aus den Einwohnermelderegistern gezogen, wovon 4236 in die Studie aufgenommen wurden. In der ersten Untersuchungswelle wurden 3021 Personen befragt, was einer Response-Rate von $71 \%$ entspricht. Ausfallgründe wa- ren am häufigsten Verweigerungen (18,2\%), gefolgt von fehlender Zeit $(3,3 \%)$ und dem Nichtantreffen der Zielperson (6,1\%). Analysen ergaben etwas höhere Verweigerungsraten bei jungen Erwachsenen über 18 Jahren und bei Frauen. 14-15jährige Jugendliche wurden, da ein Schwerpunkt der Studie in der Analyse von Frühstadien psychischer Störungen liegt, überproportional häufig in die Stichprobe aufgenommen.

\section{Erhebungsinstrument}

Die Erfassung aller Variablen erfolgte unter Anwendung der computerisierten Version (CAPI) des Münchener Composite International Diagnostic Interview (M-CIDI) [Wittchen et al., 1995; Wittchen und Pfister, 1997]. Das M-CIDI ist eine modifizierte Version des WHO-CIDI und erlaubt die standardisierte Erfassung von Symptomen, Syndromen und Diagnosen von 48 ausgewählten psychischen Störungen nach den Kriterien des DSM-IV und der ICD-10. Zusätzlich werden im Interview Informationen über den Beginn der Störung, ihre Entwicklung und Verlauf, ihre Dauer sowie störungsbedingte Beeinträchtigungen erhoben. Das MCIDI wird durch ein sogenanntes Listenheft ergänzt, welches Listen und Fragebögen enthält, die entweder mit den M-CIDI-Fragen oder zur Erfassung zusätzlicher störungsrelevanter Variablen vorgelegt werden. Die diagnostische Auswertung der M-CIDI-Angaben erfolgt unter Anwendung der M-CIDI/DSM-IV-Diagnose-Algorithmen [Pfister und Wittchen, 1995]. Im Durchschnitt dauert die Durchführung des Interviews etwa $80 \mathrm{~min}$. Reliabilität und Validität des M-CIDI wurden in mehreren Studien überprüft [vgl. hierzu Lachner et al., 1998; Wittchen, 1994; Wittchen et al., in press; Wittchen et al., submitted]. Die Ergebnisse zur Retest-Reliabilität, zu deren Bestimmung 60 Jugendliche und junge Erwachsene im Alter zwischen 14 und 24 Jahren im Abstand von durchschnittlich 38 Tagen 2 mal von unabhängigen Interviewern mit dem M-CIDI befragt wurden, verweisen auf eine sehr befriedigende Zuverlässigkeit des Instrumentes. Die prozentualen Übereinstimmungen bewegen sich zwischen 88,4 (somatoforme Störungen) und 95\% (Störungen durch Drogenkonsum, Dysthymie, bipolare Störung). Die Kappa-Koeffizienten, welche ein $\mathrm{Maß}$ für die um die Zufallswahrscheinlichkeit bereinigte Übereinstimmung von Einschätzungen auf Nominaldatenniveau darstellen, können mit Werten zwischen 0,56 (Eßstörungen) und 0,81 (Angststörungen) insgesamt als zufriedenstellend bis sehr gut bewertet werden. Speziell für somatoforme Störungen wurde für die Retest-Übereinstimmung ein Kappa-Wert von 0,62 ermittelt [Wittchen et al., in press]. Die Validität des Instrumentes wurde durch den Vergleich von klinischen Diagnosen (auf der Basis von DSM-IV-Checklisten) mit den M-CIDIDiagnosen geprüft. Für die Gruppe der somatoformen Störungen ergab sich für Übereinstimmung zwischen den klinischen Diagnosen und den M-CIDI-Diagnosen ein Kappa-Wert von 0,50. Die Sensitivität lag bei $93,8 \%$, die Spezifität bei 71,2\% [Wittchen et al., submitted].

Erfassung somatoformer Symptome und Störungen

Somatoforme Symptome und Störungen werden in der C-Sektion des MCIDI erfragt. Die Sektion beginnt mit der Vorlage einer Liste von 41 körperlichen Symptomen, für welche die befragte Person zunächst angeben soll, ob sie jemals in ihrem Leben aufgetreten sind. Anschließend wird für jedes bejahte Symptom über die Anwendung von «Prüffragen» detailliert dessen klinische Relevanz sowie die Symptomursache (körperliche Erkrankung, medikamenten-, drogen- oder alkoholbedingt) exploriert. Als klinisch relevant werden dabei Symptome bewertet, deren Ausprägung entweder zum Aufsuchen professioneller Hilfe und/oder zur mehrmaligen Medikamenteneinnahme veranlaßte, oder die wesentlich in das Leben der befragten Person eingegriffen hatten. Nur jene Symptome, die sich in dieser Exploration als klinisch relevant erweisen und nicht hinreichend durch eine körperliche Erkrankung oder eine Substanzeinwirkung erklärt werden können, werden als somatoforme Symptome diagnostiziert und zur Diagnosenbildung herangezogen.

Die M-CIDI-/DSM-IV-Diagnose-Algorithmen erlauben die Erfassung der somatoformen Schmerzstörung, Somatisierungsstörung, Konversionsstörung und Hypochondrie. Da im Rahmen unserer Symptomliste auch 
die zur Somatisierungsstörung zählenden dissoziativen Symptome (Schwächeanfälle, Bewußtlosigkeit und Amnesie) vorgelegt werden, können wir beim alleinigen Vorliegen von dissoziativen Symptomen zusätzlich ein «Dissoziatives Syndrom» NNB erfassen, das strenggenommen im DSM-IV der Gruppe der dissoziativen Störungen (und nicht der somatoformen Störungen) zugeordnet wird. Da jedoch die Konzeptualisierung der dissoziativen Störungen nicht einheitlich ist - so zählt die ICD-10 die Konversionsstörungen zu den dissoziativen Störungen - erlaubten wir uns, als «zusätzliche» somatoforme Störung eine solche dissoziative Symptomatik diagnostisch zu erfassen.

Zusätzlich kann weiter durch das M-CIDI auch die «undifferenzierte somatoforme Störung» (USD) erfaßt werden. Die Definition der USD ist unseres Erachtens jedoch zu weit gefaßt (hier genügt bereits 1 klinisch relevantes somatoformes Symptom zur Diagnosestellung), um differenziertere Analysen in der von uns untersuchten Altersgruppe durchführen zu können. Als Alternative entschlossen wir uns deshalb, als somatoformes Syndrom eine Symptomatik nach den Kriterien den SSI4,6 [Escobar et al., 1987a und b] zu prüfen. Wie oben bereits angeführt wurde, wird die Diagnose SSI4,6 gestellt, wenn Männer mindestens 4 und Frauen wenigstens 6 klinisch relevante somatoforme Symptome innerhalb eines Zeitraumes von 6 Monaten aufweisen. Eine Symptomatik nach dem SSI4,6 bezeichnen wir, da sie nicht im DSM-IV als Störungsbild operational definiert wird, im Rahmen unserer Untersuchung als somatoformes Syn- drom. Symptombilder mit somatoformen Symptomen unterhalb der geforderten Anzahl des SSI4,6 fassen wir schließlich als «undifferenziertes somatoformes/dissoziatives Syndrom» (USDS) zusammen.

In Tabelle 1 sind die zur Diagnosebildung erforderlichen Kriterien für die einzelnen Störungsbilder und Syndrome dargestellt.

\section{Interviewer/innen, Training und Durchführung der Feldarbeit}

Die Erhebungen wurden in der ersten Untersuchung (T0) von 10 in der Durchführung des M-CIDI erfahrenen klinischen Psychologen und Psychologinnen sowie von 25 professionellen Vollzeitinterviewer/innen der Infratest-Gesundheitsforschung im Alter von 26-64 Jahren durchgeführt. Die 21 Interviewerinnen und 14 Interviewer nahmen zumindest einmal an dem einwöchigen offiziellen WHO-M-CIDI-Training teil (trainiert wurde sowohl die Paper- und -Pencil-Fassung als auch die computerisierte Version) und führten mindestens 10 Übungsinterviews durch, die durch den Untersuchungsstab überprüft wurden. Kurz vor dem Beginn der Feldphase wurde ein weiteres «Auffrischungs»-Training durchgeführt.

Während der Feldarbeit wurden alle Interviewer/innen kontinuierlich von klinischen Editorinnen supervidiert. Nach jeweils maximal 10 Befragungen wurden die erhobenen Interviews einer der klinischen Editorinnen vorgelegt, die zusammen mit dem Interviewer die erhobenen Daten auf Vollständigkeit, korrekte Durchführung und Kodierung sowie auf

Tab. 1. Kriterien zur Erfassung somatoformer Störungen und Syndrome

Diagnose Erforderliche Kriterien

Somatisierungsstörung

Konversionsstörung

Dissoziative Störung NNB

SSI 4,6

Undifferenziertes somatoformes/ dissoziatives Syndrom (USDS) mindestens 8 Symptome aus 4 unterschiedlichen Symptomgruppen (s. DSM-IV)

Dauer: mindestens 2 Jahre

klinische Relevanz der Symptome (Aufsuchen medizinischer Hilfe? Mehrmalige Medikamenteneinnahme? Eingriff in das Alltagsleben?)

Symptome nicht hinreichend organmedizinisch oder durch die Wirkung einer Substanz erklärbar

mindestens 1 Konversionssymptom

klinische Relevanz der Symptomatik

Symptomatik nicht hinreichend organmedizinisch oder durch Substanzeinwirkung erklärbar Symptomatik steht in zeitlichem Zusammenhang zu einem traumatischen Erlebnis

Sorgen um eine körperliche Krankheit oder Behinderung

klinische Relevanz der Symptomatik

Sorgen nicht hinreichend organmedizinisch oder durch Substanzeinwirkung erklärbar

Sorgen führten zu mehreren ärztlichen Konsultationen/diagnostischen Abklärungen

Sorgen bleiben trotz ärztlicher Abklärung bestehen

mindestens 1 Schmerzsymptom

klinische Relevanz der Symptomatik

Symptomatik nicht hinreichend organmedizinisch oder durch Substanzeinwirkung erklärbar

mindestens 1 dissoziatives Symptom (Schwächeanfall, Bewußtlosigkeit, Amnesie)

klinische Relevanz der Symptomatik

Symptomatik nicht hinreichend organmedizinisch oder durch Substanzeinwirkung erklärbar Symptomatik steht in zeitlichem Zusammenhang zu einem traumatischen Erlebnis

Männer mindestens 4, Frauen mindestens 6 aller potentiellen somatoformen Symptome

Dauer mindestens 6 Monate

klinische Relevanz der Symptomatik

Symptomatik nicht hinreichend organmedizinisch oder durch Substanzeinwirkung erklärbar keine Diagnose bei Vorliegen einer Somatisierungsstörung

mindestens eines der potentiellen somatoformen Symptome

Dauer mindestens 6 Monate

klinische Relevanz der Symptomatik

Symptomatik nicht hinreichend organmedizinisch oder durch Substanzeinwirkung erklärbar keine Diagnose bei Vorliegen einer Somatisierungsstörung, SSI4,6, Hypochondrie, Schmerzstörung 
Tab. 2. Häufigkeit somatoformer Symptome bei 14-24jährigen Jugendlichen und jungen Erwachsenen (3021 untersuchte Personen, davon 1493 Männer und 1528 Frauen)

\begin{tabular}{|c|c|c|c|c|c|c|c|c|c|c|c|c|c|c|c|c|}
\hline \multirow[t]{3}{*}{$\begin{array}{l}\text { Anzahl der } \\
\text { Symptome }\end{array}$} & \multirow[t]{3}{*}{ Gesamt } & \multicolumn{5}{|c|}{ Männer, \% } & \multicolumn{5}{|c|}{$\begin{array}{l}\text { Frauen (einschl. gynäkol. } \\
\text { Beschwerden), \% }\end{array}$} & \multicolumn{5}{|c|}{$\begin{array}{l}\text { Frauen (gynäkol. } \\
\text { Beschwerden ausgeschlossen), \% }\end{array}$} \\
\hline & & \multicolumn{5}{|c|}{ Gesamt Alter, Jahre } & \multicolumn{5}{|c|}{ Gesamt Alter, Jahre } & \multicolumn{5}{|c|}{ Gesamt Alter, Jahre } \\
\hline & & & $14-15$ & $16-17$ & $18-21$ & $22-24$ & & $14-15$ & $16-17$ & $18-21$ & $22-24$ & & $14-15$ & $16-17$ & $18-21$ & $22-24$ \\
\hline 0 & 49,9 & 61,0 & 63,7 & 62,8 & 61,7 & 59,2 & 39,1 & 48,1 & 44,5 & 32,9 & 37,6 & 49,2 & 54,5 & 54,3 & 41,8 & 49,9 \\
\hline 1 & 26,3 & 24,2 & 24,4 & 21,7 & 23,8 & 25,1 & 28,4 & 26,8 & 26,3 & 31,8 & 27,7 & 27,6 & 25,7 & 27,5 & 33,2 & 25,4 \\
\hline 2 & 11,9 & 9,0 & 7,1 & 7,6 & 8,8 & 10,3 & 14,7 & 12,8 & 15,4 & 18,0 & 13,5 & 12,5 & 11,2 & 8,7 & 14,1 & 13,3 \\
\hline 3 & 6,6 & 4,0 & 3,6 & 6,1 & 3,8 & 3,6 & 9,2 & 5,8 & 8,5 & 9,5 & 10,3 & 6,2 & 5,7 & 6,2 & 5,9 & 6,6 \\
\hline 4 & 2,8 & 1,2 & 0,8 & 1,0 & 1,7 & 1,3 & 4,3 & 2,6 & 1,8 & 3,0 & 6,5 & 2,6 & 0,8 & 2,9 & 3,5 & 2,6 \\
\hline 5 & 1,1 & 0,2 & 0,2 & 0,6 & - & 0,2 & 2,0 & 2,3 & 1,6 & 2,1 & 1,9 & 0,7 & 0,9 & - & 0,5 & 1,0 \\
\hline 6 & 0,6 & 0,1 & 0,2 & 0,3 & - & 0,1 & 1,1 & 0,5 & 1,0 & 1,1 & 1,3 & 0,7 & 0,5 & 0,4 & 0,8 & 0,8 \\
\hline 7 & 0,4 & 0,2 & - & - & 0,3 & 0,3 & 0,7 & 0,3 & 0,4 & 1,1 & 0,7 & 0,1 & 0,7 & - & - & 0,1 \\
\hline$\geq 8$ & 0,3 & - & - & - & - & - & 0,6 & 0,9 & 0,4 & 0,5 & 0,5 & 0,3 & 0,5 & - & 0,3 & 0,4 \\
\hline 1 und mehr & 50,1 & 39,5 & 36,3 & 37,2 & 39,2 & 40,8 & 61,0 & 52,8 & 55,5 & 67,1 & 62,3 & 50,8 & 45,5 & 45,7 & 58,2 & 50,1 \\
\hline \multicolumn{6}{|c|}{ Unterschied Männer/Frauen ${ }^{1}$} & $* * *$ & $* * *$ & $* * *$ & $* * *$ & $* * *$ & $* * *$ & $* * *$ & n.s. & $* * *$ & $* * *$ & \\
\hline
\end{tabular}

1 U-Test nach Mann und Whitney, zweiseitig.

$* * * \mathrm{p}<0,001 ;$ n.s. $=$ nicht signifikant.

sonstige aufgetretene Probleme überprüfte. Registrierprobleme, vor allem im Zusammenhang mit offenen Beispielfragen sowie klinischen Aspekten, wurden an die Interviewer/innen in wöchentlichen Sitzungen rückgemeldet, um zukünftig Fehler zu vermeiden. Fehlende Angaben konnten so zeitnah zur Durchführung des Interviews nacherhoben werden.

\section{Auswertung}

Alle in den folgenden Ausführungen berichteten Prävalenzschätzungen wurden mit gewichteten Daten durchgeführt, um die an einer altersgeschichteten Stichprobe erhobenen Daten auf die Gesamtpopulation der in München lebenden 14-24jährigen beziehen zu können. Für die Abschätzung der Risikoassoziationen berechneten wir mittels logistischer Regressionen «Odds Ratios» (OR). Die Schätzungen des Erstauftretensalters von somatoformen Störungen/Syndromen wurden über Hazardraten im Rahmen der Survivalanalyse bestimmt. Abgesehen von der Survivalanalyse (SPSS für Windows, Version 7.5.2G) wurden alle Auswertungen mit SAS für Windows, Version 6.12, durchgeführt.

\section{Ergebnisse}

Wie häufig sind somatoforme Beschwerden bei Jugendlichen und jungen Erwachsenen, und welches sind die am häufigsten vorkommenden Beschwerden?

Wie aus Tabelle $2 \mathrm{zu}$ entnehmen ist, geben $50 \%$ der von uns untersuchten Jugendlichen und jungen Erwachsenen an, jemals in ihrem Leben ein klinisch relevantes, organmedizinisch, aber nicht hinreichend erklärbares Symptom gehabt zu haben. Die Hälfte hiervon berichtet über 1 somatoformes Symptom, etwa $5 \%$ über 4 oder mehr Symptome. Frauen
(61\%) geben deutlich häufiger somatoforme Beschwerden an als Männer (39,5\%; $Z=-6,46 ; p<0,001)$. Dieser Unterschied bleibt bestehen, wenn die frauenspezifischen gynäkologischen Beschwerden aus der Häufigkeitsanalyse ausgeschlossen werden. Wie Tabelle 2 ebenfalls zeigt, steigt die Prävalenz bei Männern und Frauen mit dem Alter leicht an.

Bei der Betrachtung der Art der genannten Symptome ergibt sich das in Tabelle 3 dargestellte Bild. Als die 5 häufigsten somatoformen Beschwerden werden Kopfschmerzen (11\%), Kloß im Hals (9,1\%), Bauchschmerzen (8,8\%), Unverträglichkeit von Speisen (6,5\%) sowie Schwäche- und Ohnmachtsanfälle $(5,5 \%)$ genannt. Läßt man die gynäkologischen Symptome unberücksichtigt, so zeigt sich, daß diese 5 Beschwerden sowohl von den Männern als auch von den Frauen am häufigsten genannt werden. Wie Tabelle 3 weiter zu entnehmen ist, weisen Frauen - abgesehen von den 4 Symptomen «schmerzhaftes Wasserlassen», «Schmerzen im Intimbereich», «Nahrungsmittelunverträglichkeit» und «Taubheit»bei allen Symptomen höhere Häufigkeitsraten auf als Männer.

Bei einer Betrachtung allein der gynäkologischen Beschwerden zeigt sich, daß nahezu 20\% der weiblichen Jugendlichen von schmerzhaften Regelblutungen berichten. Diese Häufigkeit wird von keinem anderen, auch nichtgynäkologischen, Symptom übertroffen. Es zeigt sich, daß schmerzhafte Regelblutungen kontinuierlich im Alter zwischen 14 und 24 Jahren zunehmen, ebenso wie unregelmäßige Menstruationszyklen, die von insgesamt 10,9\% der jungen Frauen berichtet werden. 
Tab. 3. Prävalenz spezifischer somatoformer Symptome bei Jugendlichen und jungen Erwachsenen

\begin{tabular}{|c|c|c|c|c|c|c|c|c|c|c|c|c|c|}
\hline \multirow[t]{3}{*}{ Symptom } & \multirow{3}{*}{$\begin{array}{l}\text { Gesamt } \\
\%\end{array}$} & \multicolumn{5}{|c|}{ Männer, \% } & \multicolumn{5}{|c|}{ Frauen, \% } & \multirow{3}{*}{$\begin{array}{l}\mathrm{M} / \mathrm{F}^{1} \\
\mathrm{p}\end{array}$} & \multirow{3}{*}{$\begin{array}{l}\text { Alter } \\
\mathrm{p}\end{array}$} \\
\hline & & \multicolumn{5}{|c|}{ Gesamt Alter, Jahre } & \multicolumn{5}{|c|}{ Gesamt Alter, Jahre } & & \\
\hline & & & $14-15$ & $16-17$ & $18-21$ & $22-24$ & & $14-15$ & $16-17$ & $18-21$ & $22-24$ & & \\
\hline Schmerzsymptome & 33,4 & 24,3 & & & & & 42,3 & & & & & $* * *$ & \\
\hline Bauchschmerzen & 8,8 & 6,9 & 7,1 & 4,7 & 7,2 & 7,4 & 10,6 & 9,8 & 8,8 & 13,6 & 9,9 & $* * *$ & n.s. \\
\hline Rückenschmerzen & 4,3 & 3,7 & 2,7 & 1,7 & 3,6 & 4,7 & 5,0 & 2,7 & 6,9 & 4,9 & 5,2 & n.s. & $*$ \\
\hline Gelenkschmerzen & 4,3 & 4,6 & 4,2 & 5,7 & 3,9 & 4,8 & 4,0 & 4,9 & 2,7 & 4,8 & 3,7 & n.s. & n.s. \\
\hline Schmerzen Arme/Beine & 2,0 & 1,8 & 1,5 & 2,8 & 1,6 & 1,7 & 2,2 & 2,5 & 0,7 & 3,8 & 1,6 & n.s. & n.s. \\
\hline Schmerzen Brustkorb & 3,0 & 2,3 & 1,8 & 1,8 & 2,4 & 2,7 & 3,7 & 1,8 & 2,6 & 4,3 & 4,3 & $*$ & $*$ \\
\hline Kopfschmerzen & 11,0 & 7,9 & 7,6 & 11,1 & 7,5 & 7,2 & 14,0 & 13,3 & 11,0 & 17,1 & 13,5 & $* * *$ & n.s. \\
\hline Schmerzvolle Menstruation & 9,5 & - & - & - & - & - & 18,8 & 14,0 & 16,4 & 19,8 & 20,7 & - & $*$ \\
\hline Schmerzen Wasserlassen & 0,7 & 0,8 & 1,0 & - & 1,1 & 0,9 & 0,5 & 0,5 & 0,4 & 0,9 & 0,3 & n.s. & n.s. \\
\hline Schmerzen im Intimbereich & 1,5 & 1,6 & 0,5 & 1,7 & 1,4 & 2,0 & 1,4 & 1,2 & 1,0 & 3,3 & 0,7 & n.s. & n.s. \\
\hline Andere Schmerzen & 0,3 & 0,3 & - & 0,3 & - & 0,3 & 0,4 & 0,2 & - & 0,3 & 0,6 & n.s. & n.s. \\
\hline Gastrointestinale Symptome & 13,3 & 12,1 & & & & & 14,6 & & & & & n.s. & \\
\hline Erbrechen & 2,2 & 1,2 & 1,8 & 1,4 & 0,8 & 1,2 & 3,1 & 1,6 & 1,2 & 3,0 & 4,3 & $* * *$ & n.s. \\
\hline Schwangerschaftserbrechen & 0,3 & - & - & - & - & - & 0,6 & - & - & 0,6 & 1,0 & - & n.s. \\
\hline Brechreiz & 1,7 & 1,1 & 0,7 & 1,0 & 0,7 & 1,5 & 2,3 & 0,5 & 0,7 & 3,3 & 2,9 & $*$ & $* *$ \\
\hline Durchfall & 3,3 & 3,2 & 2,0 & 2,3 & 2,3 & 4,4 & 3,3 & 2,4 & 3,9 & 2,4 & 4,0 & n.s. & $*$ \\
\hline Völlegefühl & 1,6 & 1,1 & 0,4 & 0,7 & 1,0 & 1,4 & 2,2 & 0,5 & 2,7 & 2,2 & 2,7 & $*$ & $*$ \\
\hline Unverträglichkeit Speisen & 6,5 & 6,8 & 6,7 & 5,6 & 7,1 & 7,1 & 6,2 & 6,5 & 5,9 & 6,1 & 6,3 & n.s. & n.s. \\
\hline Konversionssymptome & 6,9 & 5,1 & & & & & 8,7 & & & & & $* * *$ & \\
\hline Blindheit & 0,1 & 0,0 & - & 0,3 & - & - & 0,1 & - & - & - & 0,2 & n.s. & n.s. \\
\hline Verschwommensehen & 1,3 & 1,0 & 0,3 & 1,0 & 1,1 & 1,1 & 1,7 & 2,3 & 1,0 & 0,4 & 2,3 & n.s. & n.s. \\
\hline Taubheit & 0,2 & 0,2 & 0,2 & 0,4 & 0,2 & 0,2 & 0,1 & - & 0,3 & 0,3 & - & n.s. & n.s. \\
\hline Schwierigkeiten beim Gehen/Stehen & 0,5 & 0,4 & 0,3 & - & 0,6 & 0,5 & 0,6 & 0,3 & - & 1,8 & 0,2 & n.s. & n.s. \\
\hline Gefühlsverlust Arme/Beine & 1,4 & 1,2 & 0,8 & 0,8 & 1,1 & 1,5 & 1,5 & 1,0 & 1,3 & 2,6 & 1,3 & n.s. & n.s. \\
\hline Lähmung & 0,1 & - & - & - & - & - & 0,1 & - & - & 0,6 & - & n.s. & n.s. \\
\hline Stimmverlust & 3,7 & 2,6 & 2,3 & 4,2 & 4,0 & 1,4 & 4,8 & 6,6 & 5,1 & 4,3 & 4,3 & $* *$ & $*$ \\
\hline Krampfanfälle & 0,2 & 0,0 & 0,2 & - & - & - & 0,4 & 0,3 & - & 0,6 & 0,4 & n.s. & n.s. \\
\hline Pseudoneurologische Symptome & 9,8 & 8,7 & & & & & 10,8 & & & & & $*$ & \\
\hline Schwierigkeiten beim Wasserlassen & 0,0 & 0,1 & - & - & 0,3 & - & 0,0 & 0,1 & - & - & - & n.s. & n.s. \\
\hline Doppelbilder & 0,2 & 0,2 & 0,8 & - & - & 0,1 & 0,3 & 1,1 & - & - & 0,2 & n.s. & n.s. \\
\hline Körperliche Schwäche & 0,5 & 0,2 & 0,2 & - & 0,3 & 0,1 & 0,8 & 0,5 & 1,3 & 1,3 & 0,4 & n.s. & n.s. \\
\hline Taubheitsgefühl & 0,4 & 0,2 & - & - & - & 0,2 & 0,6 & 0,4 & 0,4 & 1,1 & 0,5 & n.s. & n.s. \\
\hline Kloß im Hals & 9,1 & 8,3 & 7,8 & 11,7 & 7,7 & 3,5 & 9,9 & 12,8 & 11,6 & 9,9 & 8,4 & n.s. & $*$ \\
\hline Dissoziative Symptome & 7,5 & 4,8 & & & & & 10,2 & & & & & $* * *$ & \\
\hline Schwäche-/Ohnmachtsanfälle & 5,5 & 3,1 & 3,4 & 2,5 & 3,0 & 3,1 & 7,9 & 5,2 & 6,3 & 8,3 & 9,0 & $* * *$ & n.s. \\
\hline Bewußtlosigkeit & 1,6 & 1,1 & 0,6 & 1,8 & 2,2 & 0,5 & 2,1 & 2,8 & 1,4 & 2,0 & 2,2 & $*$ & n.s. \\
\hline Amnesie & 0,8 & 0,8 & 0,5 & 1,7 & 1,1 & 0,4 & 0,8 & 0,9 & 1,5 & 1,2 & 0,4 & n.s. & n.s. \\
\hline Gynäkologische Symptome & 14,0 & - & & & & & 27,7 & & & & & - & \\
\hline Unregelmäßige Menstruation & 5,5 & - & - & - & - & - & 10,9 & 5,3 & 8,4 & 8,5 & 14,8 & - & $* * *$ \\
\hline Starke Regelblutungen & 3,4 & - & - & - & - & - & 6,8 & 4,0 & 7,5 & 6,3 & 7,7 & - & n.s. \\
\hline Sexuelle Symptome & 1,1 & 0,3 & & & & & 2,0 & & & & & $* * *$ & \\
\hline Sexualität wichtig & 0,7 & 0,1 & - & - & - & 0,1 & 1,4 & - & 0,4 & 1,4 & 2,1 & $* *$ & $* *$ \\
\hline Schmerzen beim Geschlechtsverkehr & 0,5 & 0,1 & - & - & - & - & 0,9 & - & 0,4 & 0,5 & 1,5 & $*$ & $*$ \\
\hline Keine Freude an sexuellen Kontakten & 0,5 & 0,1 & - & - & 0,3 & - & 0,9 & 0,3 & - & 0,6 & 1,6 & $*$ & * \\
\hline Sexuelle Probleme & 0,7 & 0,1 & - & - & - & 0,2 & 1,2 & - & - & - & 2,7 & $* *$ & n.s. \\
\hline
\end{tabular}

$* \mathrm{p}<0,05 ; * * \mathrm{p}<0,01 ; * * * \mathrm{p}<0,001$

${ }^{1} \mathrm{M} / \mathrm{F}=$ Statistischer Vergleich Männer und Frauen $\left(\chi^{2}-\right.$ Test $)$.

${ }^{2}$ Prüfung der Unterschiede zwischen den Altersgrupppen ( $\chi^{2}$ - Test). 
Abb. 1. Lifetime-Prävalenz somatoformer Störungen bei Jugendlichen und jungen Erwachsenen.

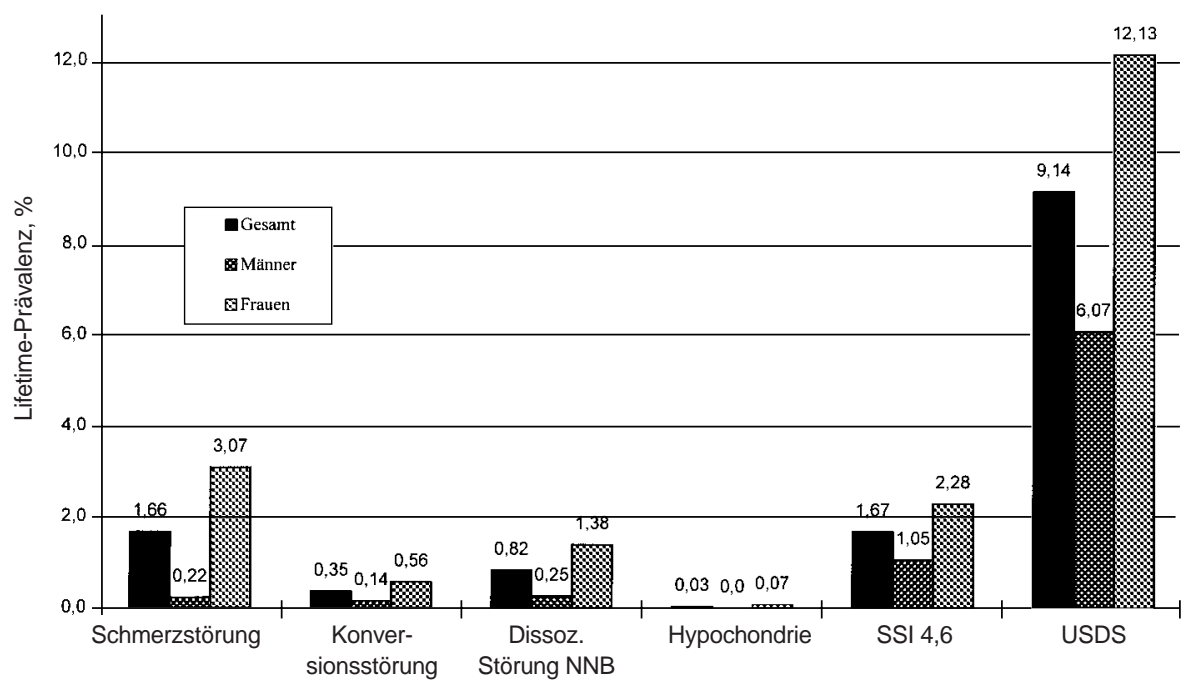

Wie häufig liegen in dieser Altersgruppe somatoforme Störungen und Syndrome vor?

In Abbildung 1 sind die Lifetime-Prävalenzen für die von uns erfaßten somatoformen Störungen und Syndrome für die Gesamtpopulation der 14-24jährigen Jugendlichen und jungen Erwachsenen dargestellt. Die Lifetime-Prävalenz gibt an, welcher Anteil der Population irgendwann einmal in ihrem Leben von einer somatoformen Störung/Syndrom betroffen war. Insgesamt wurde bei $13 \%$ der Jugendlichen und jungen Erwachsenen irgendeine somatoforme Störung/Syndrom im Verlauf des bisherigen Lebens diagnostiziert. 7,2\% der Stichprobe erfüllten die Kriterien ebenfalls in den 12 Monaten vor der Untersuchung (12-Monats-Prävalenz). Wie aus Abbildung 1 ersichtlich ist, werden somatoforme Syndrome - somit unterschwellige Störungsbilder - weit häufiger (insgesamt $10,8 \%$ ) berichtet als voll ausgeprägte Störungsbilder (insgesamt 2,7\%). Spezifische somatoforme Störungen wurden nur selten ermittelt. Keine Person erfüllt die Kriterien der Somatisierungsstörung und nur eine Person $(0,03 \%)$ die Kriterien der Hypochondrie. Als häufigste somatoforme Störung zeigt sich die Schmerzstörung (1,7\%). Wie Abbildung 1 weiter entnommen werden kann, zeigen Frauen über alle somatoformen Störungen/Syndrome hinweg doppelt so hohe Prävalenzraten wie die Männer. Die Betrachtung des Verhältnisses zwischen der Lifetime-Prävalenz und der 12-Monats-Prävalenz, welches als grobes Maß für die Persistenz einer Störung betrachtet werden kann, ergibt, daß 56\% der Personen mit einer Lifetime-Diagnose diese auch in den 12 Monaten vor der Untersuchung erfüllten.

Soziodemographische Korrelate somatoformer Störungen und Syndrome

Die Analyse soziodemographischer Korrelate somatoformer Störungen/Syndrome (Tab. 4) ergibt, daß Frauen in der von uns untersuchten Altersgruppe im Vergleich zu ihren männlichen Altergenossen ein 2,7fach höheres Risiko haben, eine der erhobenen somatoformen Störungen/Syndrome zu entwickeln. Besonders auffällig ist hierbei der ermittelte OR von 13,6 für die Schmerzstörung. Bei der Interpretation dieses Befundes sollte jedoch berücksichtigt werden, daß bei Frauen im Rahmen der Schmerzstörung auch das (von einem Fünftel der Frauen angegebene) frauenspezifische Schmerzsymptom «schmerzhafte Menstruation» gezählt wird, und sie so von vornherein eine höhere Wahrscheinlichkeit zur Diagnose einer Schmerzstörung haben.

Als weiteres nennenswertes Ergebnis ermittelten wir bei jungen Erwachsenen ab dem Alter von 18 Jahren ein gegenüber der jüngeren Altersgruppe erhöhtes Risiko, eine somatoforme Störung/Syndrom aufzuweisen. Risikoassoziationen zeigen sich ebenfalls zwischen der Diagnose des SSI4,6 und der subjektiven niedrigen oder hohen sozialen Schichtzugehörigkeit, der Diagnose eines USDS und der subjektiven Zuordnung $\mathrm{zu}$ niedrigem sozioökonomischem Status sowie der Schmerzstörung und dem Besuch der Universität. Für alle weiteren soziodemographischen Variablen ergeben sich keine bemerkenswerten Assoziationen zum Vorliegen somatoformer Störungen oder Syndrome.

\section{In welchem Alter treten somatoforme Störungen zum ersten Mal auf?}

Auf der Basis von kumulierten Hazard-Raten (Hazard-Rate = das geschätzte Risiko, in einem bestimmten Alter eine bestimmte Symptomatik zu entwickeln) zeigen sich hinsichtlich des Erkrankungsalters der erhobenen somatoformen Störungen folgende Ergebnisse:

a) Für die Schmerzstörung läßt sich ein bemerkenswerter Anstieg ab dem 12. Lebensjahr verzeichnen, der sich bis zum 17. Lebensjahr fortsetzt und dann wieder abflacht. 
Tab. 4. Demographische Korrelate spezifischer somatoformer Syndrome/Störungen

\begin{tabular}{|c|c|c|c|c|c|c|c|c|c|c|}
\hline & \multicolumn{2}{|c|}{$\begin{array}{l}\text { Irgendeine somatoforme } \\
\text { Störung/Syndrom }\end{array}$} & \multicolumn{2}{|c|}{ Schmerzstörung } & \multicolumn{2}{|c|}{$\begin{array}{l}\text { Dissoziative } \\
\text { Störung NNB }\end{array}$} & \multicolumn{2}{|c|}{ SSI4,6 } & \multicolumn{2}{|c|}{ USDS } \\
\hline & OR & $95 \% \mathrm{CI}$ & OR & $95 \% \mathrm{CI}$ & OR & $95 \% \mathrm{CI}$ & OR & $95 \% \mathrm{CI}$ & OR & $95 \%$ CI \\
\hline \multicolumn{11}{|l|}{ Alter, Jahre } \\
\hline $14-17$ & 1,00 & - & 1,00 & - & 1,00 & - & 1,00 & - & 1,00 & - \\
\hline $18-20$ & $1,60^{*}$ & $1,19-2,15$ & 0,83 & $0,38-1,81$ & 0,69 & $0,12-4,03$ & 1,47 & $0,68-3,20$ & $1,90^{*}$ & $1,34-2,69$ \\
\hline $21-24$ & $1,35^{*}$ & $1,04-1,77$ & 0,83 & $0,43-1,61$ & $3,32 *$ & $1,05-10,46$ & 1,36 & $0,68-2,71$ & 1,32 & $0,95-1,83$ \\
\hline \multicolumn{11}{|l|}{ Geschlecht ${ }^{1}$} \\
\hline Männlich & 1,00 & - & 1,00 & - & 1,00 & - & 1,00 & - & 1,00 & - \\
\hline Weiblich & $2,69 *$ & $2,13-3,40^{*}$ & 13,59 & $4,37-42,27$ & $5,01 *$ & $1,65-15,19$ & $2,20 *$ & $1,21-4,02$ & $2,05^{*}$ & $1,56-2,69$ \\
\hline \multicolumn{11}{|l|}{ Schulausbildung ${ }^{2}$} \\
\hline Haupt- & 1,26 & $0,86-1,85$ & 1,89 & $0,58-6,12$ & 0,93 & $0,14-6,03$ & 1,45 & $0,57-3,71$ & 1,18 & $0,76-1,83$ \\
\hline Real- & 0,86 & $0,61-1,22$ & 1,12 & $0,39-3,22$ & 1,16 & $0,27-4,95$ & 0,84 & $0,34-2,10$ & 0,78 & $0,52-1,17$ \\
\hline Gymnasium & 1,00 & - & 1,00 & - & 1,00 & - & 1,00 & - & 1,00 & - \\
\hline Universität & 1,01 & $0,74-1,36$ & $3,65^{*}$ & $1,53-8,70$ & 1,82 & $0,49-6,77$ & 0,97 & $0,44-2,12$ & 0,78 & $0,55-1,11$ \\
\hline \multicolumn{11}{|l|}{ Andere/ohne } \\
\hline Schulabschluß & 0,85 & $0,49-1,47$ & 2,44 & $0,63-9,42$ & 1,27 & $0,14-11,40$ & 1,58 & $0,50-4,97$ & 0,55 & $0,27-1,13$ \\
\hline \multicolumn{11}{|l|}{ Lebenssituation $^{2}$} \\
\hline Mit Eltern & 1,00 & - & 1,00 & - & 1,00 & - & 1,00 & - & 1,00 & - \\
\hline Allein & 1,16 & $0,86-1,55$ & 1,80 & $0,81-4,02$ & 0,81 & $0,29-2,24$ & 1,37 & $0,67-2,79$ & 1,10 & $0,78-1,54$ \\
\hline Mit Partner/in & 0,89 & $0,59-1,34$ & 1,77 & $0,60-4,94$ & 0,92 & $0,26-3,23$ & 0,30 & $0,06-1,51$ & 1,02 & $0,64-1,63$ \\
\hline Mit Ehegatten/in & 0,54 & $0,27-1,06$ & - & - & - & - & 0,71 & $0,14-3,59$ & - & - \\
\hline \multicolumn{11}{|c|}{ Subjektive finanzielle Situation ${ }^{2}$} \\
\hline Schlecht & $1,64^{*}$ & $1,13-2,37$ & 1,58 & $0,62-4,07$ & 0,90 & $0,18-4,49$ & 1,50 & $0,60-3,76$ & $1,56^{*}$ & $1,01-2,43$ \\
\hline In Ordnung & 1,03 & $0,79-1,33$ & 0,64 & $0,30-1,36$ & 0,96 & $0,37-2,54$ & 1,28 & $0,69-2,37$ & 1,08 & $0,79-1,47$ \\
\hline Gut & 1,00 & - & 1,00 & - & 1,00 & - & 1,00 & - & 1,00 & - \\
\hline Sehr gut & 1,09 & $0,76-1,57$ & 0,82 & $0,31-2,21$ & 1,12 & $0,30-4,19$ & 0,33 & $0,07-1,52$ & 1,35 & $0,90-2,03$ \\
\hline \multicolumn{11}{|l|}{ Soziale Schicht ${ }^{2}$} \\
\hline Niedrig & 1,25 & $0,82-1,90$ & 0,77 & $0,18-3,32$ & 2,36 & $0,71-7,89$ & $2,57^{*}$ & $1,00-6,62$ & 1,04 & $0,62-1,74$ \\
\hline Mittel & 1,00 & - & 1,00 & - & 1,00 & - & 1,00 & - & 1,00 & - \\
\hline Hoch & $1,53^{*}$ & $1,21-1,93$ & 1,67 & $0,92-3,04$ & 1,29 & $0,51-3,23$ & $2,71^{*}$ & $1,49-4,94$ & 1,31 & $0,99-1,72$ \\
\hline \multicolumn{11}{|l|}{ Urbanizität $^{2}$} \\
\hline München-Stadt & 1,08 & $0,83-1,40$ & 1,36 & $0,65-2,86$ & 1,29 & $0,45-3,75$ & 1,06 & $0,55-2,06$ & 0,99 & $0,73-1,33$ \\
\hline München-Land & 1,00 & - & 1,00 & - & 1,00 & - & 1,00 & - & 1,00 & - \\
\hline $\begin{array}{l}\text { OR = Odds-Ratio } \\
1 \text { Alter kontrollie } \\
2 \text { Alter und Gesch } \\
* \mathrm{p}<0,05 .\end{array}$ & Konfid & $\begin{array}{l}\text { azintervall. } \\
\text { ert. }\end{array}$ & & & & & & & & \\
\hline
\end{tabular}

Diese Zeit kann für die untersuchte Altersgruppe der 14-24jährigen demnach als Hochrisikozeit für die Entwicklung einer Schmerzstörung begriffen werden, wobei auch hier bei der Interpretation die Bedeutung von Menstruationsschmerzen bei weiblichen Jugendlichen ab der Pubertät in Betracht gezogen werden sollte.

b) Die Konversionsstörung und die Dissoziative Störung NNB zeigen erst ab dem 16./17. Lebensjahr einen bemerkbaren Anstieg.

Bei den von uns erfaßten somatoformen Syndromen hingegen läßt sich bereits ab dem 5./6. Lebensjahr ein Anstieg nachweisen, wobei beim SSI4,6 ab dem 19. Lebensjahr, hingegen beim USDS ab dem 22. Lebensjahr keine weitere Zunahme mehr zu verzeichnen ist (d.h. keine neuen Fälle mehr hinzukommen).

Welche anderen Störungen treten häufig zusätzlich zu somatoformen Störungen auf (Komorbidität)?

Wie aus Tabelle $5 \mathrm{zu}$ entnehmen ist, erfüllt ein großer Anteil der Jugendlichen mit somatoformen Störungen/Syndromen auch die Kriterien für eine oder mehrere psychische Störungen. Die niedrigste Komorbiditätsrate wurde für das USDS hier erfüllen 33,6\% der Personen die Kriterien mindestens einer weiteren psychischen Störung - und die höchste Rate für die Konversionsstörung (75,8\% der Personen mit einer Konversionsstörung zeigen mindestens eine komorbide psychi- 
Tab. 5. Komorbidität zwischen somatoformen Störungen/Syndromen und anderen psychischen Störungen (gewichtet in $\%$ )

\begin{tabular}{|c|c|c|c|c|c|}
\hline $\begin{array}{l}\text { Somatoforme } \\
\text { Störungen/Syndrome }\end{array}$ & $\begin{array}{l}\text { Ohne komorbide } \\
\text { Störung }\end{array}$ & + 1 Störung & + 2 Störungen & + 3 Störungen & + 4 Störungen \\
\hline \multicolumn{6}{|l|}{ Schmerzstörung } \\
\hline Gesamt & 51,7 & 23,7 & 15,4 & 9,2 & - \\
\hline 14-17 Jahre & 79,6 & 14,3 & 6,0 & - & - \\
\hline 18-24 Jahre & 37,0 & 28,6 & 20,3 & 14,1 & - \\
\hline \multicolumn{6}{|l|}{ Konversionsstörung } \\
\hline Gesamt & 24,2 & 39,9 & 26,3 & 9,7 & - \\
\hline 14-17 Jahre & 80,8 & 19,2 & - & - & - \\
\hline 18-21 Jahre & - & 48,7 & 37,5 & 13,8 & - \\
\hline \multicolumn{6}{|c|}{ Dissoziative Störung NNB } \\
\hline Gesamt & 50,5 & 38,4 & 7,7 & 3,5 & \\
\hline 14-17 Jahre & 69,9 & 30,1 & - & - & - \\
\hline 18-24 Jahre & 46,9 & 39,9 & 9,1 & 4,1 & - \\
\hline \multicolumn{6}{|l|}{ SSI 4, 6} \\
\hline Gesamt & 35,9 & 22,0 & 19,0 & 13,6 & 2,1 \\
\hline 14-17 Jahre & 46,6 & 9,7 & 43,8 & - & - \\
\hline 18-24 Jahre & 32,5 & 25,9 & 11,1 & 17,9 & 2,8 \\
\hline \multicolumn{6}{|l|}{$U S D S$} \\
\hline Gesamt & 66,4 & 20,9 & 9,7 & 2,2 & 0,8 \\
\hline 14-17 Jahre & 72,1 & 22,7 & 4,2 & 1,0 & - \\
\hline 18-24 Jahre & 64,7 & 20,4 & 11,3 & 2,6 & 1,1 \\
\hline
\end{tabular}

Als komorbide Störungen wurden Substanzstörungen, affektive Störungen, Angst- und Eßstörungen betrachtet. sche Störung) ermittelt. Über alle somatoformen Störungsbilder hinweg nimmt die Komorbidität mit dem Alter zu, ebenso die Anzahl an komorbiden Störungen.

Somatoforme Störungen/Syndrome sind in der von uns untersuchten Altersgruppe am häufigsten mit affektiven Störungen assoziiert. So weisen über $40 \%$ der Jugendlichen und jungen Erwachsenen mit einer Schmerzstörung, Konversionsstörung oder SSI4,6-Symptomatik zusätzlich die Diagnose einer affektiven Störung auf, und sie zeigen im Vergleich zur Gesamtpopulation der in der Basisuntersuchung befragten 14-24jährigen Jugendlichen und jungen Erwachsenen ein über 4mal höheres Risiko, diese Diagnose zu erhalten (Schmerzstörung: $\mathrm{OR}=4,03, \mathrm{p}<0,05$; Konversionsstörung: $\mathrm{OR}=4,61, \mathrm{p}<0,05$; SSI4,6: OR = 4,76, p < 0,05). Ebenfalls bemerkenswert sind die ermittelten hohen Risiken für die Konversionsstörung $(\mathrm{OR}=27,5 ; \mathrm{p}<0,05)$, die Dissoziative Störung NNB $(\mathrm{OR}=$ 11,1; $<<0,05)$ und die SSI4,6-Symptomatik $(\mathrm{OR}=10,99 ; \mathrm{p}<$ $0,05)$, zusätzlich eine Eßstörung aufzuweisen. Bei den letztgenannten Risiken muß allerdings einschränkend angemerkt werden, daß sich die Werte innerhalb großer Vetrauensintervalle bewegen.

Signifikant höhere Risiken, eine Angststörung aufzuweisen, ergeben sich für den SSI4,6 (OR = 4,57; $\mathrm{p}<0,05)$, die Schmerzsstörung $(\mathrm{OR}=2,84 ; \mathrm{p}<0,05)$ und die USDS $(\mathrm{OR}=$ $1,53 ; \mathrm{p}<0,05)$. Bedeutsame Assoziationen zu Substanzstörungen zeigen lediglich der SSI4,6 (Alkoholstörung: $\mathrm{OR}=2,14$; Störung durch illegale Substanzen: 3,85 , alle $p<0,05)$ und die Dissoziative Störung NNB (Störung durch illegale Drogen: $\mathrm{OR}=4,09 ; \mathrm{p}<0,05)$.
Beeinträchtigungen und Aufsuchen professioneller Hilfe bei Personen mit somatoformen Störungen/Syndromen

Um Hinweise auf die aktuelle Beeinträchtigung der Jugendlichen aufgrund psychischer und körperlicher Probleme zu erhalten, wurden folgende Angaben erhoben: a) die Anzahl an Tagen, an denen sie während der letzten 4 Wochen aufgrund psychischer Probleme überhaupt nicht oder nur eingeschränkt in der Lage waren, ihren normalen Alltagsaktivitäten (Schule, Arbeit, Haushalt, Studium) nachzugehen, und b) die Anzahl an Tagen, an denen sie wegen körperlicher Probleme keine Alltagsaktivitäten ausführen konnten.

In Tabelle 6 finden sich für unterschiedliche «somatoforme» Diagnosegruppen die prozentualen Anteile an beeinträchtigten Personen. Es zeigt sich, daß im Vergleich zu Personen ohne DSM-IV-Diagnose(n) diejenigen mit somatoformen Störungen/Syndromen häufiger entweder überhaupt nicht oder aber nur eingeschränkt in der Lage sind, wegen psychischer Probleme ihren Alltagsaktivitäten nachzugehen. So berichten 4,2\% der Personen mit somatoformen Störungen/Syndromen, aber nur 1\% derjenigen ohne DSM-IV-Diagnose, in den letzten 4 Wochen mindestens einen Tag nicht fähig zur Ausübung von Alltagsaktivitäten gewesen zu sein ( $\mathrm{OR}=4,48$; $\mathrm{p}<0,05)$. Etwa $20 \%$ der Betroffenen waren mindestens einen Tag lang in ihren Alltagsaktivitäten eingeschränkt (Personen ohne Diagnose: 5,1\%; OR $=4,1 ; \mathrm{p}<0,05)$. Wie Tabelle 6 ebenfalls zu entnehmen ist, zeigen vor allem Personen mit einer somatoformen Störung und einer komorbiden psychischen Störung ein höheres Risiko, im Alltag wegen psychi- 


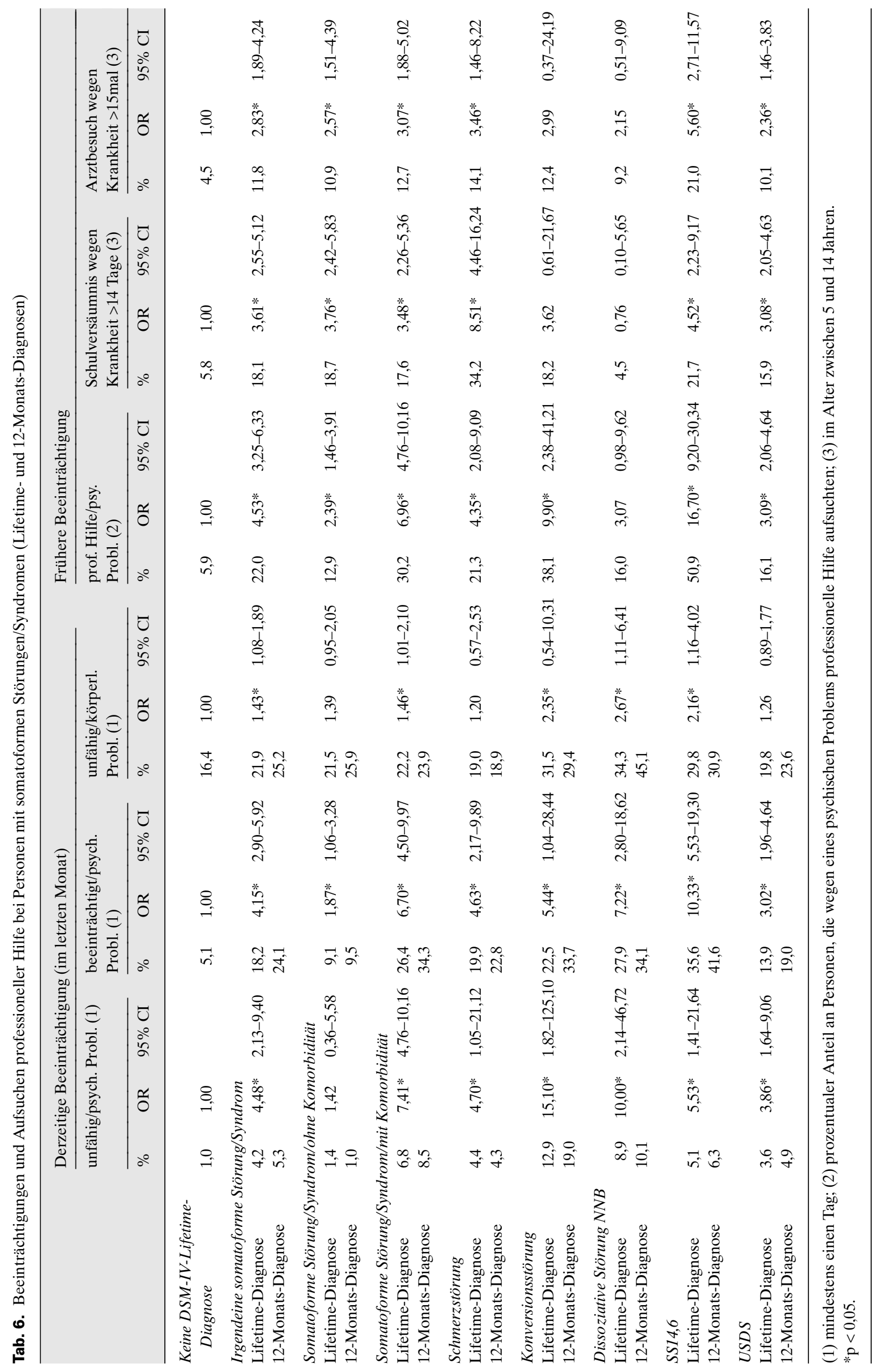


scher Probleme beeinträchtigt zu sein. Bei der Betrachtung von spezifischen somatoformen Beschwerdenbildern sind die höchsten Beeinträchtigungswerte für Personen mit einer Konversionsstörung (unfähig/Alltagsaktivitäten: $\mathrm{OR}=15,1 ; \mathrm{p}$ $<0,05)$ und diejenigen mit einer Symptomatik nach dem SSI4,6 (eingeschränkt/Alltagsaktivitäten: OR = 10,3; $\mathrm{p}<0,05$ ) nachzuweisen. Betroffene mit somatoformen Störungen/Syndromen sind im Vergleich $\mathrm{zu}$ denjenigen ohne DSM-IVStörung ebenfalls häufiger aufgrund körperlicher Probleme eingeschränkt (21,9 vs. $16,4 \%$; $\mathrm{OR}=1,43 ; \mathrm{p}<0,05$ ).

In Tabelle 6 finden sich ebenfalls Angaben hinsichtlich früherer Beeinträchtigungen sowie über das Aufsuchen professioneller Hilfe von Personen mit somatoformen Störungen/Syndromen. Wie sich zeigt, weisen Betroffene mit somatoformen Störungen (mit oder ohne komorbide Störungen) im Vergleich zu Personen ohne eine DSM-IV-Diagnose bereits in der Kindheit (im Alter zwischen 5 und 14 Jahren) Beeinträchtigungen auf. So berichten sie etwa $3 \mathrm{mal}$ so häufig wie Personen ohne DSM-IV-Diagnose (18,1 vs. 5,8\%; OR = 3,61; $\mathrm{p}<$ 0,05), durchschnittlich im Jahr mindestens 14 Tage die Schule wegen Krankheit versäumt zu haben. Ebenfalls häufiger als Personen ohne DSM-IV-Diagnosen haben diejenigen mit somatoformen Störungen/Syndromen wegen psychischer (22,0 vs. 5,9\%; OR = 4,53; $\mathrm{p}<0,05)$ und auch wegen körperlicher Probleme $(11,8$ vs. $4,5 \%$; OR $=2,83 ; \mathrm{p}<0,05)$ professionelle Hilfe aufgesucht.

\section{Diskussion}

Basierend auf einer prospektiven epidemiologischen Längsschnittuntersuchung, in welcher unter anderem auch somatoforme Störungen und Syndrome nach dem DSM-IV erhoben wurden, werden epidemiologische Befunde zur Verbreitung dieser Störungen bei Jugendlichen und jungen Erwachsenen vorgelegt. Im Rahmen dieser Basisuntersuchung wurden 3021 14-24jährige Jugendliche und junge Erwachsene aus München-Stadt und -Land befragt. Die Aussagekraft der Befunde kann als hinreichend gewertet werden, da die Erhebungsmethodik (persönliche Interviews, spezielle befragungs- und auswertungstechnische Merkmale des M-CIDI) eine gute Reliabilität, Validität und Auswertungsobjektivität aufweist [Lachner et al., 1998]. Ziel des Artikels war es, zunächst die Prävalenz von somatoformen Symptomen, Syndromen und Störungen in dieser Altersgruppe zu beschreiben. Des weiteren sollte beantwortet werden, in welchem Alter sich somatoforme Störungen/Syndrome zum ersten Mal zeigen, ob sie im Verbund mit anderen psychischen Störungen auftreten und wie beeinträchtigend sie sind. Nach unserem Wissen können mit der vorliegenden Arbeit erstmals verläßliche Angaben darüber gemacht werden, ob somatoforme Beschwerden und Störungen im Jugend- und jungen Erwachsenenalter von Bedeutung sind.

Bei der Betrachtung der Symptomebene ergab sich, daß etwa die Hälfte der Personen in der Altersgruppe zwischen 14 und 24 Jahren die Erfahrung einer somatoformen Beschwerde aufweist. Frauen sind häufiger betroffen als Männer, eine Aussage, die Garber et al. [1991] ebenfalls für Schulkinder treffen konnten und die sich auch mit an Erwachsenenstichproben ermittelten Befunden deckt [Escobar et al., 1987a]. Kopf- und Bauchschmerzen gehören zu den am häufigsten genannten Beschwerden. Dieses Ergebnis wird durch die Literatur bestätigt [Zusammenf. s. Campo und Fritsch, 1994]. Obwohl einzelne somatoforme Beschwerden relativ häufig von Jugendlichen und jungen Erwachsenen berichtet werden, erfüllt nur ein geringer Anteil (2,7\%) auf die gesamte Lebenszeit bezogen die diagnostischen Kriterien für eine somatoforme Störung nach dem DSM-IV. Verglichen mit anderen in unserer Studie erfaßten Störungsbildern (Lifetime-Prävalenz für eine affektive Störung: 16,7\%; für eine Angststörung: $14,1 \%$ ) treten voll ausgeprägte somatoforme Störungen in der Altersgruppe der 14-24jährigen eher selten auf. Hieraus ließe sich zunächst der Schluß ziehen, daß somatoformen Störungen in diesem Alter noch keine wesentliche Bedeutung zukommt. Bei der Betrachtung von unterschwelligen somatoformen Symptombildern ergibt sich allerdings ein anderes Bild: Nahezu 11\% der Jugendlichen und jungen Erwachsenen berichten nun, irgendwann in ihrem Leben an einem somatoformen Syndrom gelitten zu haben. Vergleichbar zu den einleitend erwähnten Befunden zur Häufigkeit des SSI4,6 [Escobar et al., 1989] erhöht sich die Prävalenz somit merklich bei Erweiterung der diagnostischen Kriterien. Zieht man in Betracht, daß zur Diagnosestellung dieser unterschwelligen Störungsbilder die klinische Relevanz der Symptome und die Persistenz der Symptomatik von mindestens 6 Monaten vorausgesetzt werden, so berichtet ein beachtlicher Anteil der Jugendlichen und jungen Erwachsenen von einer nach unserer Einschätzung nicht vernachlässigbaren Symptomatik Dies könnte ein Hinweis darauf sein, daß weniger restriktive Diagnosekriterien adäquater somatoforme Störungen in jüngeren Altersgruppen erfassen können.

Weiterhin weisen Jugendliche und junge Erwachsene sowohl mit ausgeprägten somatoformen Störungen als auch mit unterschwelligen Beschwerdenbildern zusätzlich häufig noch andere psychische Störungen und auch nennenswerte Beeinträchtigungen im psychosozialen Bereich auf, eine Aussage, die mehreren, an Erwachsenenstichproben ermittelten Befunden entspricht [Escobar et al., 1987a; 1987b; Kirmayer und Robins, 1991; Swartz et al., 1986]. Diese Ergebnisse können als Hinweise für die Validität der in der Studie herangezogenen diagnostischen Kategorien somatoformer Beschwerdenbilder gewertet werden. Es muß jedoch angemerkt werden, daß sie lediglich Assoziationen darstellen, d.h., wir wissen nichts über deren zeitliche Zusammenhänge. Um über die Richtung der Zusammenhänge nicht ausschließlich zu spekulieren, muß in zukünftigen Analysen im Detail aufgeschlüsselt werden, welche zeitlichen Muster sich für die ermittelten Assoziationen in der befragten Altersgruppe zeigen. 
In der vorliegenden Arbeit untersuchten wir somatoforme Symptomkonstellationen, wie sie vom DSM-IV vorgegeben werden. Das Spektrum somatoformer Störungen wurde aufgrund der einleitend erwähnten theoretischen Erwägungen um die Dissoziative Störung NNB, SSI4,6 und das USDS erweitert, alles Symptomkonstellationen, die unter strenger Anwendung des DSM-IV der dort angeführten USD zuzuordnen wären. Validitätshinweise für die erfaßten diagnostischen Kategorien entnahmen wir den Komorbiditäts- und Beeinträchtigungsbefunden. Eine Beurteilung der Validität der herangezogenen diagnostischen Kategorien schließt jedoch auch die Frage ein, ob sich die a priori vorgegebenen Symptomkonstellationen auch empirisch in den untersuchten Altersgruppen wiederfinden. Weitere diesen Punkt untersuchende Analysen werden wir als nächsten Schritt durchführen, um abschätzen zu können, inwieweit die durch das DSM-IV vorgegebenen Symptomgruppierungen als valide für die «wahre» Symptomatik in der untersuchten Altersgruppe betrachtet werden können.

Ungeachtet der eben gestellten Validitätsfragen wird auch noch die Frage beantwortet werden müssen, ob die DSM-IVoder empirisch ermittelten Symptomkonstellationen spezifische Krankheitsbilder (im Sinne nosologischer Entitäten) darstellen oder ob sie in der untersuchten Altergruppe nicht vielmehr Ausdruck einer unspezifischen Vulnerabilität sind und ihnen eventuell eine Schlüsselbedeutung in der Pathogenese anderer psychischer Störungen, wie z.B. Depressionen oder Angststörungen, zukommt. Die im Titel gestellte Frage nach der Existenz somatoformer Störungen bei Jugendlichen und jugendlichen Erwachsenen können wir somit letztlich nicht hinreichend befriedigend beantworten. Unsere Ergebnisse zeigen, daß die vom DSM-IV vorgegebenen Störungskategorien in der von uns untersuchten Population in den oben angegebenen Häufigkeiten diagnostiziert werden können. $\mathrm{Ob}$ sie in der von uns untersuchten Altersgruppe allerdings (a) als eigenständige Krankheitseinheiten, (b) als Vulnerabilitätsmarker oder Prodromalphasen in der Entwicklung anderer psychischer Störungen oder (c) lediglich als körperliche Begleiterscheinungen einer bestimmten Entwicklungsphase, die sich durch starke körperliche Veränderungen auszeichnet, aufzufassen sind, können wir anhand der Befunde der Basisuntersuchung nicht beantworten. Auf der Grundlage des prospektiven Designs bietet unsere Studie jedoch die Möglichkeit, diese kritischen Punkte bei Vorliegen der Längsschnittdaten näher zu beleuchten. Wir hoffen, spezifischere Antworten dann nicht nur auf die Frage, ob es somatoforme Störungen bei Jugendlichen und jungen Erwachsenen gibt, sondern auch auf die Frage nach der klinischen Eigenständigkeit dieser Beschwerdenbilder geben zu können.

\section{Literatur}

American Psychiatric Association: Diagnostic and Statistical Manual of Mental Disorders; 3rd ed. Washington DC, Author, 1980.

American Psychiatric Association: Diagnostic and Statistical Manual of Mental Disorders; 4th ed. Washington DC, Author, 1994.

Bass C, Murphy M: Somatoform and personality disorders: Syndromal comorbidity and overlapping developmental pathways. J Psychosom Res 1995; 39:403427.

Campo J, Fritsch S: Somatization in children and adolescents. J Am Child Adolesc Psychiatry 1994;33:12231235 .

Deighton CM, Nicol AR: Abnormal illness behaviour in young women in a primary care setting: Is Briquet's syndrome a useful category? Psychol Med 1985;15: 515-520.

Escobar JI, Burnam MA, Karno M, Forsythe A, Golding JM: Somatization in the community. Arch Gen Psychiatry 1987a;44:713-718.

Escobar JI, Canino G: Unexplained physical complaints. Psychopathological and epidemiological correlates. Br J Psychiatry 1989;154:24-27.

Escobar JI, Golding JM, Hough RL, Karno M, Burnman MA, Wells KB: Somatization in the community: Relationship to disability and use of services. Am J Public Health 1987b;77:837-840.

Escobar JI, Rubio-Stipec M, Canino G, Karno M: Somatic symptom index (SSI): A new and abridged somatization construct. J Nerv Mental Dis 1989;177:140146.
Fydrich T, Schmitz B: Komorbidität bei Personen mit Somatisierungsstörung. Vortrag auf dem 39. Kongress der Deutschen Gesellschaft für Psychologie, 25.-29. September 1994, Hamburg.

Garber J, Walker LS, Zeman J: Somatization symptoms in a community sample of children and adolescents: Further validation of the Children's Somatization Inventory. J Consult Clin Psychol 1991;3:588-595. Katon W, Lin E, von Korff M, Russo J, Lipscomb P, Bush T: Somatization: A spectrum of severity. Am J Psychiatry 1991;148:34-40.

Kellner R: Functional somatic symptoms and hypochondriasis. Arch Gen Psychiatry 1985;42:821-833.

Kirmayer LJ, Robbins JM: Three forms of somatization in primary care: Prevalence, co-occurrence, and sociodemogaphic characteristics. J Nerv Ment Dis 1991; 179:647-655.

Lachner G, Wittchen H-U, Perkonigg A, Holly A Schuster P, Wunderlich U, Türk D, Garczynski E, Pfister H: Structure, content and reliability of the Munich Composite International Diagnostic Interview (M-CIDI). Substance use sections. Eur Addict Res 1998;4: 28-41.

Pfeiffer E, Schröder G, Lehmkuhl U: Somatoforme Störungen bei Kindern und Jugendlichen. Psychotherapie 1997;2:102-105.

Pfister H, Wittchen H-U: M-CIDI Computerprogramm. München, Max-Planck-Institut für Psychiatrie, 1995.

Regier DA, Boyd JH, Burke JD Jr, Rae DS, Myers JK, Kramer M, Robins LN, George LK, Karno M, Locke BZ: One month prevalence of mental disorders in the United States. Arch Gen Psychiatry 1988;45:977-986.
Regier DA, Narrow WE, Rae DS, Manderscheid RW, Locke BZ, Goodwin FK: The de facto US mental and addictive disorders service system. Epidemiologic Catchment Area prospective 1-year prevalence rates of disorders and services. Arch Gen Psychiatry 1993; 50:85-94.

Rief W, Hiller W: Somatoforme Störungen. Körperliche Symptome ohne organische Ursachen. Bern, Huber, 1992.

Rief W, Schaefer S, Hiller W, Fichter MM: Lifetime diagnoses in patients with somatoform disorders: Which came first? Eur Arch Clin Neurosci, 1992; 241: 236-240.

Robins LN, Locke BZ, Regier DA: An overview of psychiatric disorders in America; in: Robins LN, Regier DA (eds): Psychiatric Disorders in America. The Epidemiologic Catchment Area Study. New York, The Free Press, 1991, pp 328-366.

Swartz M, Blazer D, George L, Landerman R: Somatization in a community population. Am J Psychiatry 1986;143:1403-1408.

Tomasson K, Kent S, Coryell W: Comparison of four diagnostic systems for the diagnosis of somatization disorders. Acta Psychiatr Scand 1991;88:311-315.

Üstün TB, Sartorius N (eds): Mental Illness in General Health Care: An International Study. New York, Wiley, 1995.

Viederman M: Somatoform and factitious disorders. In: Michels R, et al. (eds): Psychiatry. Philadelphia, Lippincott, 1993, pp 1-20. 
Wittchen H-U: Reliability and validity studies of the WHO-Composite International Diagnostic Interview (CIDI): A critical review. J Psychiatr Res 1994;28: 57-84.

Wittchen H-U, Beloch E, Garczynski E, Holly A Lachner G, Perkonigg A, Pfütze E-M, Schuster P, Vodermaier A, Vossen A, Wunderlich U, Zieglgänsberger S: a) Münchener Composite International Diagnostic Interview (M-CIDI, Paper-pencil 2.2, 2/95). München, Max-Planck-Institut für Psychiatrie, Klinisches Institut (Eigendruck), 1995.

Wittchen H-U, Essau CA, von Zerssen D, Krieg CJ, Zaudig M: Lifetime and six-month prevalence of mental disorders in the Munich Follow-up Study. Eur Arch Psychiatry Clin Neurosci 1992;241:247-258.
Wittchen H-U, Gander F, Pfister H, Steiger A, Trenkwalder C, Sonntag A, Hundt W: To what degree the Composite International Diagnostic Interview (CIDI) correctly identifies DSM-IV disorders? Testing validity issues in a clinical sample. J Psychiatr Res (submitted).

Wittchen H-U, Lachner G, Wunderlich U, Pfister H: Test-retest reliability of the computerized DSM-IV version of the Munich Composite International Diagnostic Interview (M-CIDI). Soc Psychiatry Psychiatr Epidemiol (in press).

Wittchen H-U, Perkonigg A, Lachner G, Nelson CB: The early developmental stages of psychopathology study (EDSP) - objectives and design. Eur Addict Res 1998;4:18-27.
Wittchen H-U, Pfister H (eds): DIA-X-Interviews: Manual für Screening-Verfahren und Interview; Interviewheft Längsschnittuntersuchung (DIA-X-lifetime); Ergänzungsheft (DIA-X-Lifetime); Interviewheft Querschnittuntersuchung (DIA-X-12 Monate); Ergänzungsheft (DIA-X-12Monate); PC-Programm zur Durchführung des Interviews (Längs- und Querschnittuntersuchung); Auswertungsprogramm. Frankfurt, Swets und Zeitlinger, 1997.

World Health Organization: International Classification of Disease (ICD-10). Geneva, World Health Organisation Division of Mental Health, 1991.

World Health Organization: Composite International Diagnostic Interview: Core version 1.1. Geneva, World Health Organization, 1992.

World Health Organization: Tenth revision of the international classification of diseases. ICD-10. Chapter V (F). Mental and behavioral disorders. Diagnostic criteria for research. Geneva, World Health Organization, 1993. 